\title{
Controlled Neural Response Generation by Given Dia- logue Acts Based on Label-aware Adversarial Learning
}

\author{
Seiya Kawano \\ Koichiro Yoshino \\ Satoshi Nakamura
}

Nara Institute of Science and Technology

seiya.kawano@iken.jp

Guardian Robot Project (GRP), Institute of Physical and Chemical Research (RIKEN)

koichiro.yoshino@riken.jp

Nara Institute of Science and Technology

s-nakamura@is.naist.jp

keywords: dialogue system, dialogue act, neural conversation model, conditional response generation, reinforcement learning

\begin{abstract}
Summary
Building a controllable neural conversation model (NCM) is an important task. In this paper, we focus on controlling the responses of NCMs using dialogue act labels of responses as conditions. We introduce a reinforcement learning framework involving adversarial learning for conditional response generation. Our proposed method has a new label-aware objective that encourages the generation of discriminative responses by the given dialogue act label while maintaining the naturalness of the generated responses. We compared the proposed method with conventional methods that generate conditional responses. The experimental results showed that our proposed method has higher controllability conditioned by the dialogue acts even though it has higher or comparable naturalness to the conventional models.
\end{abstract}

\section{Introduction}

Neural conversation models (NCMs), which learn a direct mapping between a dialogue history and a response utterance based on neural networks, are widely researched as an approach for building non-task-oriented dialogue systems [Serban 16, Vinyals 15]. However, its response generation process is entirely black-boxed, unlike classical dialogue systems, making it difficult to interpret why the model generates a response to a dialogue context. This problem complicates transferring the technology of NCMs to practical situations, such as introducing a chat-bot into business scenes. Introducing a mechanism for controlling the response generation of NCMs by clear intention helps to solve this problem.

Conventionally, dialogue act labels have been exploited to control the intention of response generation in both taskand non-task-oriented dialogue systems [Meguro 10, Shibata 16, Yoshino 15]. A dialogue act is defined as a unit that reveals the functions of associated turns in dialogues [Jurafsky 97]. Dialogue act labels are unique classes to distinguish the dialogue acts of given utterances (e.g., "Hello! can be abstracted to dialogue act label "Greeting") [Boyer 10, Bunt 12]. The dialogue acts clarify the roles of utter- ances in the dialogue context. In pragmatics, a speech act is also known as a similar classification standard to a dialogue act, an essential component to analyze and model both human-human and human-machine dialogues [Alston 00]. However, the benefits of dialogue act labels have not been exploited enough in NCMs, although studies of classical dialogue systems focus on modeling dialogues based on interpretable units, such as dialogue act labels. This inability limits the effectiveness of NCMs in several points. First, having interpretable system intentions by dialogue act labels enables humans to understand the behavior of dialogue systems. Second, modeling the high-level decision-making policy apart from response generation will enable the system to respond with a well-organized and thoughtful response. For example, as shown in Figure 1, the required response to the system is often different even the recent dialogue context is the same. In such a case, the high-level decision by the dialogue manager is important, which uses other information such as user preference.

Some existing studies have tackled this kind of prob", lem for controlling responses of NCMs using actual labels (e.g., dialogue acts, emotions, persona); however, these works still suffer from limitations [Huang 18, Li 16b, Wen 


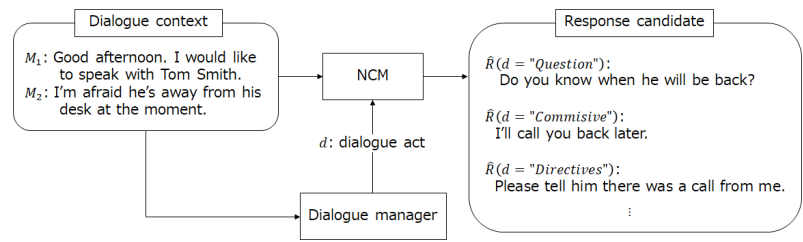

Fig. 1 Response generation conditioned by dialogue act label: the NCM generates responses that represent dialogue act labels determined by a high-level decision-making model such as a dialogue manager.

15]. One crucial issue is that they do not have any explicit training objectives to guarantee that a generation has discriminability $^{* 1}$ to the given condition. In other words, to enforce the conditional generation of an NCM, we have to establish a new training objective which we call a labelaware objective.

To solve this problem, we introduce an extended framework of sequential generative adversarial networks [Yu 17] to improve the controllability of NCMs, given a dialogue act label as the condition. We propose a label-aware adversarial learning framework that alternatively trains both a generator, which creates a response to the given dialogue act label, and a discriminator, whose objective is to evaluate both the naturalness and the discriminability. The discriminator evaluates the validity of the generated responses by a classification model: label awareness. The evaluation results are used as a reward of reinforcement learning for training the generator. The method to control the response generation by adversarial learning is proposed by [Kawano 19]. We generalized the method from the viewpoint to control the generator by reinforcement learning.

In this paper, we first describe the task of conditional response generation by dialogue act labels and existing approaches (Chapter 3). We introduce a reinforcement learning framework involving adversarial learning to address the problem of conditional response generation (Chapter 4). In experiments, we performed automatic and human evaluation of the controllability and naturalness of the generated responses (Chapter 5). The experimental results showed that our proposed model significantly improved the controllability scores in both automatic and human subjective evaluations, even it achieved better or comparable naturalness to existing methods (Chapter 6). We discuss the challenges for the advancement of conditional response generation given dialogue act labels in neural conversation models by analyzing our experimental results

\footnotetext{
*1 Note that we use "discriminative" and "discriminability" as terms referring to whether a given dialogue act label is represented in response utterances.
}

(Chapter 7).

\section{Related Work}

Dialogue systems that have dialogue management modules determine a dialogue act or a frame of a system response using statistical methods, such as reinforcement learning [Keizer 17, Meguro 10, Yoshino 15, Young 10]. Their response generation modules create responses according to these dialogue acts or frames based on rules, templates, agendas, or other statistical models [Oh 00, Xu 00]. In recent years, neural network-based generation modules have been widely used for building response generation modules [Dušek 18]. The controllability of neural generation systems is a big research issue.

One work proposed a conditional language model called Semantically Conditioned Long Short-Term Memory (SCLSTM) [Wen 15], which generates utterances based on dialogue act labels and frames in the domain of restaurant navigation dialogues using a gating mechanism. Furthermore, several improved SC-LSTM models are proposed in E2E NLG Challenge [Dušek 18]. However, their training framework requires state frames that entirely express the function and the contents of the target utterances. It is unrealistic to apply this method to building a non-taskoriented dialogue system. Another work proposed a neural conversation model (NCM) based on a conditional variational autoencoder [Zhao 17], which generates responses with high diversity at the discourse-level using latent variables considering dialogue acts. They suggest that the dialogue act is key information to improve the quality of NCM responses. This indication contributes to our motivation to control the response generation of NCMs based on the dialogue act labels. However, since this model focuses on learning well-organized latent variables using dialogue act labels, its training objective does not focus on improving the discriminability of generated responses by dialogue act labels.

There is another research trend for controlling NCMs with a given condition, such as persona or emotion labels [Huang 18, Li 16b]. These NCMs are optimized by softmax cross-entropy loss (SCE-loss), which calculates losses word-by-word. However, such existing training objectives do not necessarily guarantee that the generated response has high discriminability to the given class label. SCE-loss can optimize the word prediction itself, but it cannot evaluate whether the property of the generated responses belongs to the given classes. To address this problem, some works incorporate an auxiliary classifier to generative models, including NCMs, to improve the dis- 
criminability of their generated sentences $[\mathrm{Hu} 17$, Shen 20, Zhou 18b]. In order to feedback the evaluation signals of the auxiliary classifier to the generative model, the problem of discrete sampling from the generative model needs to be addressed. REINFORCE algorithm based on reinforcement learning [Shen 20, Williams 92, Zhou 18b] or sampling from a multinomial distribution parametrized using softmax function [Jang 16, Hu 17] are widely used to address this problem. They achieved the back-propagation between the auxiliary classifier and the generative model. However, their approaches must cope with over-fitting to the auxiliary classifier because the classifier is naively trained from the training data.

In this paper, we introduce an extended framework of sequential generative adversarial networks [Li 17a, Tuan 19, Yu 17]. Different from methods incorporating with static auxiliary classifiers, our framework alternatively trains both a generator, which creates responses according to a given dialogue act label and a discriminator, which has an objective to evaluate both the naturalness and the discriminability of the generated responses by the given condition. Our architecture prevents the over-fitting problem to the auxiliary classifier by dynamically updating the discriminator through adversarial learning. Our architecture also makes it possible to consider the total quality of the generated responses, unlike SCE-loss, which is optimized for each term.

\section{Conditional Response Generation by Given Dialogue Act Labels}

In this paper, we focus on controlling a conditional neural conversation model (NCM) using dialogue act labels. We assume a non-task-oriented dialogue as the target domain. In this chapter, we first address the conditional response generation task tackled in this paper (Section 3 1). Then we describe the conventional architecture of a conditional response generation model based on minimizing softmax cross-entropy loss (Section 3·2).

\subsection{Task Settings}

The task we focus on is building a controllable NCM by a given condition, typically a dialogue act label. The problem is defined as generating the response word sequence $\hat{R}=\left[\hat{w}_{1}, \hat{w}_{2}, \cdots, \hat{w}_{T^{\prime}}\right]$ given dialogue history $M=\left[M_{1}\right.$, $\left.M_{2}, \ldots, M_{N}\right]$ and of response dialogue act label $d \in \mathcal{D}$. Here $N$ is the length of the dialogue history, $T^{\prime}$ is the number of words in the response, and $\mathcal{D}$ is the set of dialogue act labels. As shown in Figure 1, response $\hat{R}$ has to satisfy not only the behavioral characteristics of a given dialogue act label but also appropriateness in the dialogue context (=history).

We assume that a dialogue act label is decided by a highlevel decision-making model, such as a dialogue manager, built separately from the NCM. This allows us to connect high-level decision-making models to the NCM freely, and we may enable to provide a useful generalization and a personalization to users. We only focus on the problem of whether NCMs can generate responses according to given dialogue act labels, since building the decision-making model is beyond the scope of this paper. We use oracle dialogue act labels in a corpus when we evaluate response generation systems.

\subsection{General Conditional Neural Conversation Model with Dialogue Act Labels}

We introduce a general conditional NCM controlled by given dialogue act labels as the baseline. We built a conditional NCM based on a hierarchical encoder-decoder model, which explicitly uses dialogue act labels in its decoding steps (Figure 2). We adopt vector concatenation between a word vector and a vectorized condition to feed decoder input as a widely used method of conditional NCMs [Huang 18, Li 16b].

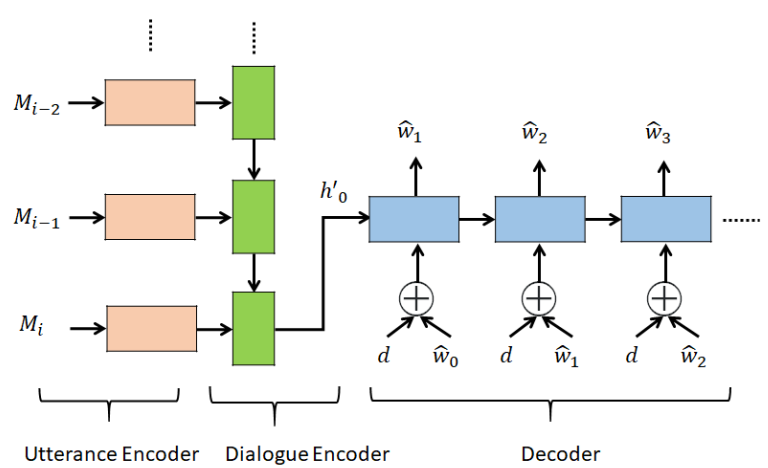

Fig. 2 Conditional NCM with dialogue act labels

The encoder network has a hierarchical structure that consists of utterance and dialogue encoders [Serban 16]. The utterance encoder receives a word at each time step using forward RNNs to encode the utterance into a fixedlength vector:

$$
h_{i, t}=\mathrm{RNN}_{\text {utterance }}\left(h_{i, t-1}, \operatorname{Embed}\left(w_{i, t}\right)\right) .
$$

Here $i$ is the number of turns in the dialogue context, and $h_{i, t}$ is the hidden vector obtained by inputting each word $w_{i, t}$ in utterance $M_{i}$. The embedding layer (Embed) projects $w_{i, t}$ to a fixed-length word vector to be used as input.

Utterance vectors are given to the dialogue encoder: 


$$
c_{i}=\mathrm{RNN}_{\text {context }}\left(c_{i-1}, h_{i, T_{i}}\right) .
$$

Here $T_{i}$ is the number of words in the utterance $M_{i}$, and $h_{i, T_{i}}$ is a hidden vector obtained at the last step of the utterance encoder. Resultant vector $c_{N}$ is fed to the decoder and used as initial hidden state $h_{0}^{\prime}$ to generate a response sentence. In the decoder, hidden state $h_{t}^{\prime}$ of the decoder and the output probability of word $p_{t}$ are calculated:

$$
\begin{aligned}
h_{t}^{\prime} & =\operatorname{RNN}_{\mathrm{dec}}\left(h_{t-1}^{\prime},\left[\operatorname{Embed}\left(\hat{w}_{t-1}\right) ; \operatorname{Embed}_{\mathrm{da}}(d)\right]\right), \\
p_{t} & =\operatorname{Softmax}\left(\operatorname{Linear}_{\text {proj }}\left(h_{t}^{\prime}\right)\right) .
\end{aligned}
$$

Here Linear ${ }_{\text {proj }}$ is a projection layer, which maps $h_{t}^{\prime}$ to a vector of vocabulary size $|\mathcal{V}|$. Embed $_{\mathrm{da}}$ is a linear transformation layer that converts target dialogue act label $d$ into a fixed-length vector. $\hat{w}_{t}$ is sampled from a probabilistic distribution $p_{t} \in \mathbb{R}^{|\mathcal{V}|}$ obtained by Softmax function from the output of projection layer, and used as a part of the input of the next time step.

In this decoding architecture, we expect the decoding result to correspond to both the given dialogue act label and the dialogue history by inputting dialogue act label $d$ in addition to the already generated words. $d$ is encoded to a fixed-length vector using an embedding layer $\left(\operatorname{Embed}_{\mathrm{da}}\right)$ as input.

In general, such NCMs are trained by minimizing the softmax cross-entropy loss (SCE-loss):

$$
L_{\mathrm{SCE}}=-\sum_{t=1}^{T} \log p_{t}\left(w_{t}\right) .
$$

Here $T$ is the number of words in the ground-truth response $R=\left[w_{1}, w_{2}, \cdots, w_{T}\right]$, and $p_{t}\left(w_{t}\right)$ denotes the value that corresponds to the ground-truth word $w_{t}$ in the probabilistic distribution $p_{t}$ obtained from Softmax function from the output of projection layer.

SCE-loss optimizes the word prediction at each decoding step. However, it does not explicitly consider the given dialogue act label in the loss calculation during training. It only considers the likelihood of ground-truth words. Related to this, the NCM optimized by SCE often generates biased responses that contain frequently used words [ $\mathrm{Li}$ 16a]. This problem will be critical in situations where the NCM must strictly represent a given dialogue act label. It is because dialogue acts of individual utterances change depending on dialogue context even if they have the same literal meaning (or contain frequent words representing the given dialogue act's characteristics). Due to such complexity of human language, SCE-Loss, which does not consider the whole structure of the generated response, may not handle the differences in dialogue acts appropriately. In the following chapter, we address this problem by introducing an explicit training objective based on reinforcement learning involving adversarial learning for generating well-controlled responses by dialogue act labels.

\section{Enhancing Conditional Response Gener- ation Based on Reinforcement Learning}

Conditional generation based on SCE-loss does not guarantee that the generation result follows a given condition. We introduce an objective function based on a framework of reinforcement learning (RL) that enhances the performance of the conditional response generation. This is because RL-based systems have the advantage of high flexibility in the design of reward functions, including the auxiliary classifier compared to other approaches [Hu 17]. Our RL-based system optimizes the conditional NCM by maximizing the reward, which evaluates whether the generated response obeys the given dialogue act label. In other words, we optimize the conditional NCM with guarantees that responses are generated according to a given dialogue act label. In the most current system, the auxiliary classifier is used to give rewards [Zhou 18b]. However, since using a static classifier caused an over-fitting problem of the generator, we prevent this problem by introducing adversarial learning, which dynamically changes the classifier based on the generation results from the generator.

In this chapter, we explore different types of approaches for optimizing conditional NCMs. We first formulate a conventional optimization framework for a case with a static dialogue act classifier for RL and address its problem (Section 4.1 and Section 4.2). We extend this optimization framework based on the sequential generative adversarial network (SeqGAN) [Li 17a, Yu 17], including a different classifier and a dynamically changed classifier named a discriminator, for improving both the controllability and the naturalness of the conditional response generation (Section $4 \cdot 3$ ).

\subsection{REINFORCE Algorithm for Conditional Response Generation}

We introduce a policy gradient (REINFORCE Algorithm) [Williams 92], which is a direct differentiation of the reinforcement learning (RL) objective. The problem of response generation in NCMs is defined as generating response word sequence $\hat{R}=\left[\hat{w}_{1}, \hat{w}_{2}, \cdots, \hat{w}_{T^{\prime}}\right]$ given dialogue context $M$. Such a word selection process is defined as a word-selecting action sequence, which is generated by an actual policy in a Markov decision process (MDP) [Li 17a, Ranzato 16]. We define a reward func- 
tion based on the classifier to evaluate the validity of conditional response generation in NCMs. The evaluation score is fed as a reward to optimize the generator' s policy by maximizing the expected reward of the generated responses. The gradient of the objective function is defined $^{* 2}$ :

$$
\begin{aligned}
\nabla J_{\mathrm{RL}}(\theta) \simeq & \frac{1}{T^{\prime}} \sum_{t=1}^{T^{\prime}} \sum_{\hat{w}_{t} \in \mathcal{V}} Q_{D_{\phi}}^{G_{\theta}}\left(\hat{R}_{1: t-1}, \hat{w}_{t}, d\right) \\
& \cdot \nabla_{\theta} G_{\theta}\left(\hat{w}_{t} \mid \hat{R}_{1: t-1}, d\right) \\
= & \frac{1}{T^{\prime}} \sum_{t=1}^{T^{\prime}} \mathbb{E}_{\hat{w}_{t} \sim G_{\theta}}\left[Q_{D_{\phi}}^{G_{\theta}}\left(\hat{R}_{1: t-1}, \hat{w}_{t}, d\right)\right. \\
& \left.\cdot \nabla_{\theta} \log p_{\theta}\left(\hat{w}_{t} \mid \hat{R}_{1: t-1}, d\right)\right] .
\end{aligned}
$$

Here $\theta$ is the parameters of the policy. $\mathcal{V}$ is a vocabulary, $\hat{R}_{1: t-1}$ indicates the already generated word sequence, and $d$ is the dialogue act label used as a condition (state in MDP). $p_{\theta}\left(\hat{w}_{t} \mid \hat{R}_{1: t-1}\right)=G_{\theta}\left(\hat{w}_{t} \mid \hat{R}_{1: t-1}, d\right)$ is the generative probability of word $\hat{w}_{t} \in \mathcal{V}$ (action in MDP) in the decoder. $Q_{D_{\phi}}^{G_{\theta}}\left(\hat{R}_{1: t-1}, \hat{w}_{t}, d\right)$ is an action-value function that gives an expected future reward of word-generating action $\hat{w}_{t}$ given the state: already generated word sequence $\hat{R}_{1: t-1}$ and dialogue act label $d$. The expectation $\mathbb{E}[\cdot]$ can be approximated by sampling.

To evaluate the action-values for intermediate states, the Monte Carlo search under the policy of $G_{\theta}$ is applied to sample the future words. Each search ends until the end of word of response is sampled, or the sampled response reaches the maximum length. To obtain a stable reward and reduce the variance, we use an $N$-time Monte Carlo $\operatorname{search}^{* 3}$ [Yu 17] as:

$$
\left\{\hat{R}_{1: T_{1}}^{1}, \cdots, \hat{R}_{1: T_{N}}^{N}\right\}=\operatorname{MC}^{G_{\theta}}\left(\hat{R}_{1: t}, d ; N\right) .
$$

Here $T_{n}$ denotes the number of words in the response sampled by the $n$-th Monte Carlo search. $\left(\hat{R}_{1: t}, d\right)$ is the current state and $\left\{\hat{R}_{1: T_{1}}^{1}, \cdots, \hat{R}_{1: T_{N}}^{N}\right\}$ are sampled from the policy $G_{\theta}$. The reward function provides $N$ rewards for the sampled $N$ responses, respectively. The final reward for the intermediate state is calculated as the average of the $N$ rewards. Therefore, we calculate the reward in timestep $t$ for the generated response with the length $T^{\prime}$, as:

$$
\begin{aligned}
& Q_{D_{\phi}}^{G_{\theta}}\left(\hat{R}_{1: t-1}, \hat{w}_{t}, d\right)= \\
& \begin{cases}\frac{1}{N} \sum_{n=1}^{N} D_{\phi}\left(\hat{R}_{1: T_{n}}^{n}, M, d\right) & \text { for } t<T^{\prime} \\
D_{\phi}\left(\hat{R}_{1: T}, M, d\right) & \text { for } t=T^{\prime} .\end{cases}
\end{aligned}
$$

*2 Note that we followed the derivation and notations shown in [Yu 17].

*3 We set $N=5$. However, since the computation cost of the Monte Carlo search is high when training the large model, we can also adopt an approach for speeding up the training, such as REGS [Li 17a].
Here $D_{\phi}(\cdot)$ is a reward function given by a classifier, which has a parameter $\phi$. The classifier evaluates the validity of the generated responses to the given condition $d$. We introduce different classifiers to update the response generation model.

\section{$4 \cdot 2$ Static Reward Function Based on Dialogue Act Clas- sifier}

We first introduce a naive reward function using a static dialogue act classifier. This is inspired by previous works that introduced an auxiliary classifier to encourage conditional generation [Shen 20, Zhou 18b]. The auxiliary classifier predicts whether the generated responses follow the given condition with a multi-class classifier. In other words, it forces NCM's response generation to follow a given condition, such as the dialogue act label. We built classifier $D_{\phi}$ based on a hierarchical encoder to predict the dialogue act label of generated response $\hat{R}$ (Figure 3 ).

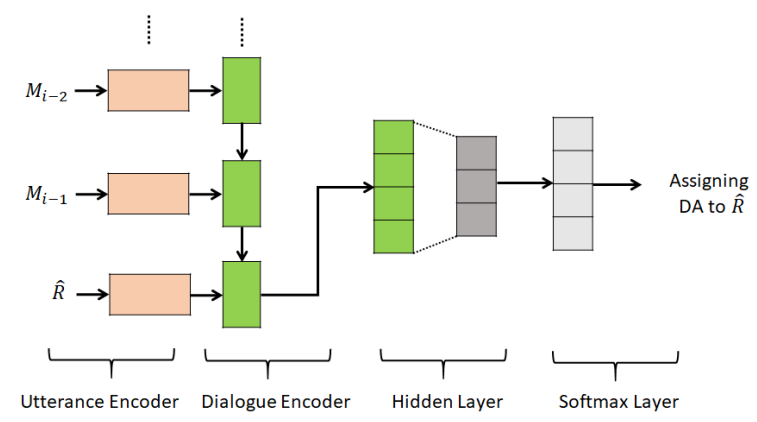

Fig. 3 Dialogue act classifier with hierarchical encoder

Dialogue history $M$ and generated responses $\hat{R}$ are encoded to contextual representation $c$ by the hierarchical encoder. We use the same encoder structure of the conditional NCM formulated in Eqs. (1)-(2) for encoding the dialogue history and the generated response. Contextual representation $c$ is fed into a fully connected layer to classify the dialogue act of response $\hat{R}$ :

$$
\begin{aligned}
& h_{\mathrm{da}}=\operatorname{MLP}(c), \\
& p_{\mathrm{da}}=\operatorname{Softmax}\left(\operatorname{Linear}_{\mathrm{da}}\left(h_{\mathrm{da}}\right)\right) .
\end{aligned}
$$

Here MLP is a multi-layer perceptron that applies the Relu function to each hidden layer, and Linear ${ }_{\mathrm{da}}$ is a linear transformation layer, which transforms $h_{\mathrm{da}}$ to a vector of a number of unique dialogue act labels. $p_{\mathrm{da}}$ is the classification probability of the dialogue act of $\hat{R}$ to class $d$.

We use posterior probability $p_{\mathrm{da}}(d \mid \hat{R}, M)$ estimated by the classifier as the reward of the generator. It gives higher rewards if the posterior probability to given dialogue class $d$ is high. We expect that this classifier will encourage 
training the generator to make controlled responses according to the given dialogue act labels. However, it may cause a problem where the generator is over-fitted to the dialogue act classifier; when using a classifier that is naively trained by maximum likelihood estimation, the conditional NCM invariably learns a "lazy" policy and chooses the easiest way to represent a given dialogue act. This choice will improve the controllability, but it will not lead to natural responses. To prevent such problems, optimization based on SCE-loss as pre-training must be incorporated.

\subsection{SeqGAN for Conditional Response Generation with Dialogue Acts}

We introduce the extended framework of the sequential generative adversarial network (SeqGAN) [Yu 17]. SeqGAN uses a discriminator instead of a classifier, which dynamically updates the evaluation system of the generation results. Our framework alternatively trains both the generator, which generates a response according to a given dialogue act label, and the discriminator, which has an objective that evaluates both the naturalness and the discriminability of a generated response for a given dialogue act label. A benefit of such a training strategy is that the system can dynamically update the parameters for further iteratively improving the generative model while avoiding any over-fitting to evaluation system; discriminator $D_{\phi}$. It can penalize a response that is unnatural or typical, even if the generated response represents the given dialogue act label. [Kawano 19] proposed this system to use adversarial learning for controlling the generator. We regard the system as an extension of a framework based on a static reward function described in the previous section.

In our framework, we incorporated dialogue act labels in the discriminator in a general SeqGAN (Figure 4). We propose two discriminators for incorporating dialogue act label information in the discriminator: implicit and explicit. We also propose an ensemble approach that integrates both discriminators. Each method is described below in respective sections.

\section{$\S 1$ Binary Objective: Implicit-Discriminator}

We first propose an implementation of the discriminator that incorporates dialogue acts in its additional feature vectors. We use the same structure of the dialogue act classifier described in Section 4.2 and apply a concatenated vector between contextual representation $c$ and the embedding vector of the dialogue act label as the MLP input. We also change the dimension of the output layer to 2 . We call this architecture "implicit" and define its objective function:

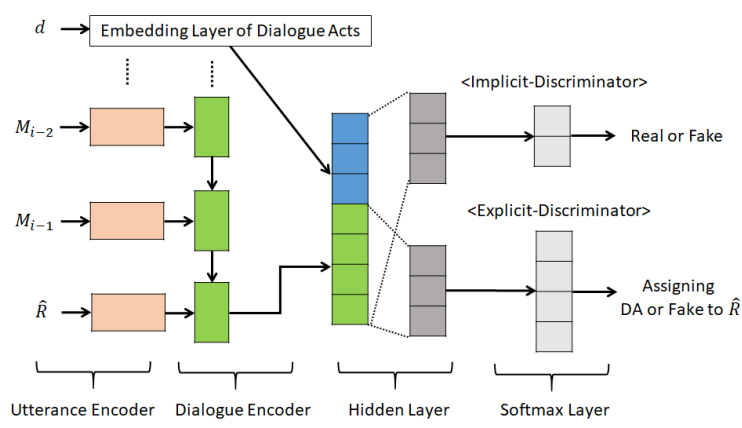

Fig. 4 Implicit \& Explicit-Discriminator

$$
\begin{aligned}
& \min _{\phi}-\mathbb{E}_{R \sim p_{\text {data }}(\cdot \mid M, d)}\left[\log D_{\phi}(R, M, d)\right] \\
& -\mathbb{E}_{\hat{R} \sim G_{\theta}(\cdot \mid M, d)}\left[\log \left(1-D_{\phi}(\hat{R}, M, d)\right)\right] .
\end{aligned}
$$

Here $p_{\text {data }}$ is the probability distribution of actual response $R$ on the training data. $D_{\phi}(\hat{R}, M, d)$ is the probability of response $\hat{R}$ belonging to the binary classes: an actual response in the training data (real) or a generated response (fake).

We expect that the implicit discriminator will use the information of the dialogue acts as a feature and discriminate the generated results as fakes if they do not follow a given dialogue act label (Figure 4, upper-right). Some works have similar approaches in emotional response generation [Kong 19, Sun 18]. However, this discriminator remains a simple extension of the standard discriminator, which classifies responses in two classes. In other words, since the objective is not changed, it probably struggles to distinguish the class (dialogue act label) of the responses. We introduce another discriminator to solve this problem in the next section.

\section{§ 2 Multi-class Objective: Explicit-Discriminator}

We propose an approach that extends the classification problem of the discriminator from the binary classification of fake/real to multi-class classification that distinguishes target dialogue act classes (Figure 4 lower-right). It also applies an additional class to the static dialogue act classifier. This discriminator has a multi-class objective for $|\mathcal{D}|+1$ class classification. Here $|\mathcal{D}|$ is the number of unique dialogue act labels; another one is a fake class for categorizing the responses as generated. We call this architecture "explicit" and define its objective function:

$$
\begin{array}{r}
\min _{\phi}-\sum_{i=1}^{|\mathcal{D}|} \mathbb{E}_{R \sim p_{\text {data }}(\cdot \mid M, d)}\left[\log D_{\phi}(d=i \mid R, M)\right] \\
-\mathbb{E}_{\hat{R} \sim G_{\theta}(\cdot \mid M, d)}\left[\log D_{\phi}\left(d_{\text {fake }} \mid \hat{R}, M\right)\right] .
\end{array}
$$

We used posterior probability $D_{\phi}(d \mid \hat{R}, M)$ estimated by the discriminator as the generator' $\mathrm{s}$ reward. We ex- 
pect that this discriminator will encourage generator training to provide discriminative sentences with dialogue acts because the generation results are penalized if they are not natural and fail to follow the manners of the given dialogue acts. Similar approaches, which use multi-class objectives in GAN, have been proposed for image generation [Odena 16, Salimans 16]. These works suggested that multi-class objectives are useful for stable training on a small amount of training data.

\section{§3 Ensemble Objective: Ensemble-Discriminator}

[Kawano 19] indicated that the implicit-discriminator generally focuses more strictly on classifying real or fake, whereas the explicit-discriminator tends to focus on classifying dialogue act labels. However, the explicit-discriminator' s properties resemble those of the static dialogue act classifier if the training is over-fitted. The explicit discriminator will cause the same over-fitting problem to the classifier, if it always provides a fixed amount of fake probability.

Thus, we propose an ensemble approach that combines the properties of both the implicit-discriminator and the explicit-discriminator ( $D_{\phi^{\text {imp }}}$ and $D_{\phi^{\text {exp }}}$ ) for stable training. We define the reward function using the harmonic-mean:

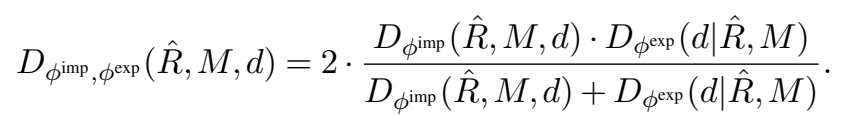

This reward function $D_{\phi^{\text {imp }}, \phi^{\exp }}$ simultaneously optimizes both (12)-(13). This is expected to ensure that the NCM does not learn the biased policy by the characteristics of one or the other discriminator.

\section{\$ 4 Training Procedure}

The training procedure of a conditional NCM with SeqGAN is shown in Algorithm 1.

First, we pre-train the conditional NCM by minimizing the SCE-loss $\left(L_{\mathrm{SCE}}\right)$. Then we apply alternate training between the generator and the discriminator. In G-steps for updating the generator, we sample generated response $\hat{R}$ with dialogue history $M$ and dialogue act label $d$ then calculate $Q_{D_{\phi}}^{G_{\theta}}$ by discriminator $D_{\phi}$. We update the parameters of $G_{\theta}$ using the calculated $Q_{D_{\phi}}^{G_{\theta}}$. Here we perform the update by $L_{\mathrm{CE}}$ after updating by RL to stabilize the training. This approach works as teacher-forcing and prevents the collapse of policies due to the model' $s$ inability to access the reference response [Li 17a]. In D-steps for updating the discriminator, we sample real response $R$, given dialogue history $M$ and dialogue act label $d$ from the training data, and then sample fake response $\hat{R}$ from generator $G_{\theta}$. We update the parameters of discriminator $D_{\phi}$ using the real and fake samples. Note that when we apply the

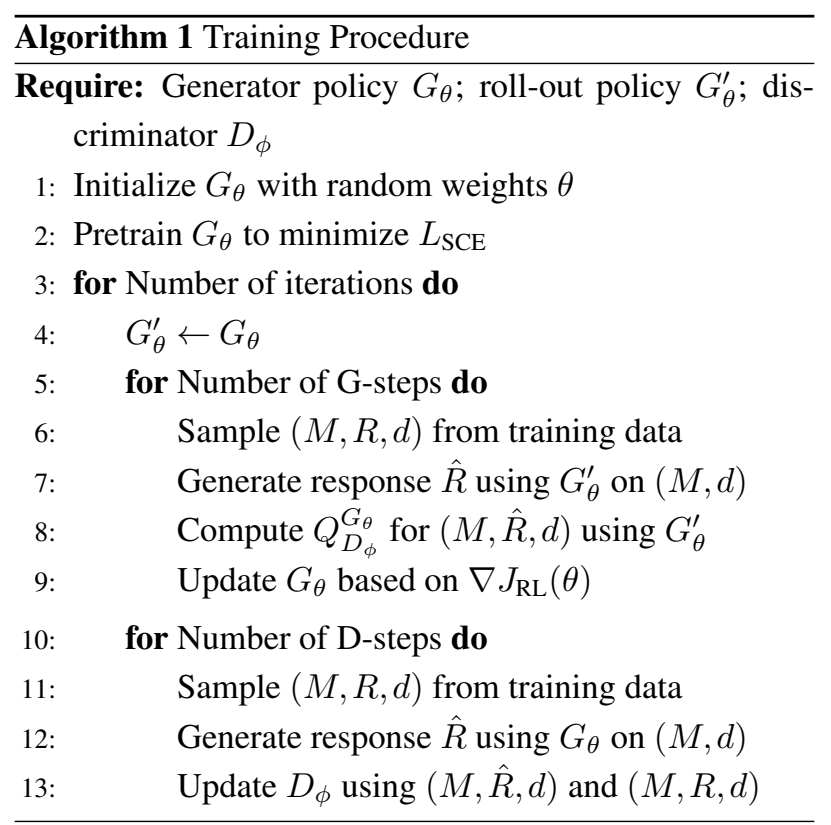

naive RL-based method presented in Section $4 \cdot 2$, the system does not use D-steps. Instead, a pre-trained classifier of dialogue act classes is used as the discriminator.

\section{Experimental Settings}

We experimentally investigated the advantages of the proposed controlled response generation system of both its controllability and naturalness. We performed both automatic and human evaluations. In this chapter, we describe their experimental settings.

\section{$5 \cdot 1$ Dataset}

We used the DailyDialog corpus [Li 17b] and the Switchboard dialogue act corpus [Jurafsky 97] for the evaluations. The DailyDialog corpus consists of ten categories from a wide variety of topics. We used training/validation/test sets of the DailyDialog (Table 1). This corpus is annotated with four dialogue act labels for each utterance: inform, questions, directives, and commissive.

Table 1 Number of dialogues/utterances in DailyDialog

\begin{tabular}{c||c|c}
\hline & Dialogues & Utterances \\
\hline \hline Train & 11,118 & 76,052 \\
\hline Validation & 1,000 & 7,069 \\
\hline Test & 1,000 & 7,740 \\
\hline
\end{tabular}

The switchboard dialogue act corpus (SWBD), which is a large-scale corpus containing telephone speech [Jurafsky 97, Stolcke 00], is annotated with the SWBD-DAMSL tagset, which consists of 42 dialogue act labels (Table 2). We also used this dataset for the evaluations. Since SWBD is a conversation with more than one utterance per turn, unlike 
the DailyDialog, we added to the suffix special symbols that indicate the type of conversation floor in each utterance [Zhao 17].

Table 2 Number of dialogues/utterances in SWBD

\begin{tabular}{c||c|c}
\hline & Dialogues & Utterances \\
\hline \hline Train & 2,316 & 20,788 \\
\hline Validation & 60 & 5,255 \\
\hline Test & 62 & 5,481 \\
\hline
\end{tabular}

\section{$5 \cdot 2$ Competing Models}

We compare the different kind of training objectives and neural conversation models.

\section{$\S 1$ Model Settings}

Based on a hierarchical encoder, we used the following types of NCMs as baselines:

- ASEQ2SEQ: a standard neural conversation model that encodes a previous utterance as a query for decoding a response with an attention mechanism (generalattention) [Luong 15].

- HED: a hierarchical encoder-decoder model [Serban 16] without conditioning to a decoder described in Section $3 \cdot 2$.

- CHED: a HED model with a conditioning mechanism. We gave the condition (dialogue act labels) described in Section 3.2.

We can also use other advanced networks for neural conversation generation [Peng 19, Zhou 18a]. However, note that we focus on training objectives to enhance the generation performance of the NCMs.

\section{$\$ 2$ Training Objectives}

We trained these NCMs with the following training objectives:

- w/SCE: optimization by minimizing the softmax crossentropy loss (SCE-loss) described in Section 3.2.

- w/ KgCVAE: optimization by knowledge-guided conditional variational autoencoder (KgCVAE) [Zhao 17]. We used CHED for the basic network, and we did not use any meta-features except for dialogue acts. The generation performance depends "heavily" on randomness due to sampling from a latent variable, thus we calculate the average results of five trials when we input an oracle dialogue act.

- w/ AC: joint optimization with auxiliary classifier. Unlike RL-based approaches, we use sampling from a multinomial distribution parametrized by softmax function for receiving the feed-back from auxiliary classifier. We basically follow the algorithm described in [Hu 17]. We use CHED as the generator network, and the dialogue act classifier described in Section $4 \cdot 2$ as the auxiliary classifier. Here, we removed objective function and network related to VAE according to their official implementation ${ }^{* 4}$, since the training of latent variable is not the scope of this paper.

- w/ RL: optimization by the static reward function based on the dialogue act classifier described in Section 4.2.

- w/ IMPLICIT: optimization by SeqGAN with the implicit-discriminator described in Section $4 \cdot 3 \cdot 1$.

- w/ EXPLICIT: optimization by SeqGAN with the implicit-discriminator described in Section 4.3.2.

- w/ ENSEMBLE: optimization by SeqGAN with the implicit and explicit-discriminator described in Section $4 \cdot 3 \cdot 3$.

\section{§3 Training Settings}

We used the same hyper-parameter settings in these NCMs and classifiers. The vocabulary size was 15000 , the word embedding size was 300 , the dialogue act embedding size was 50 , and the hidden vector size was 300 . We used a two-layer Gated Recurrent Unit (GRU) [Cho 14] as an RNN. In training, we used a mini-batch size of 64 , a Gstep of 1, a D-step of 5, and an Adam optimizer [Kingma $14]$ with a learning rate of $1 e-5$. We used teacher-forcing in the training process [Vinyals 15]. We used the model with the highest controllability for the generated response and the deterioration of the perplexity within 1.0 points from the pre-training model on the validation set.

\subsection{Automatic Evaluation Metrics}

We automatically evaluated the generation results using references in the test set. We used a beam search (a beamwidth of 5) for generating examples for evaluation ${ }^{* 5}$. For automatic evaluations, we used the following seven metrics:

- Perplexity (PPL) is a general metric for evaluating a language model performance. The model likelihoods of the reference responses are calculated. Note that the perplexity scores do not directly reflect the generation quality; for example, dull responses also have good perplexity scores.

- ROUGE, which is a popular automatic evaluation metric of language generation tasks, calculates the similarity between references and generated responses [Lin 04] based on n-gram recall. We used ROUGE-L, which is a variant of ROUGE. ROUGE-L compares the references and the generated responses based on the longest common sub-sequences between them.

- Vector Pooling calculates the cosine similarity between the reference and generated response vectors

*4 https://github.com/asyml/texar/tree/master/examples/text_style_transfer

$* 5$ We used the top-1 response from the beam search candidates. 
[Tao 17]. Each sentence vector is calculated by concatenating both the max and the min-pooling vectors of the word vectors in a sentence. This is a simple extension of vector extrema [Liu 16], which is widely used in dialogue evaluations.

- NN Scorer is a neural network-based scorer that measures the relatedness between the generated response and its dialogue context [Tao 17]. The scorer is trained by the negative samples of response pairs and randomly selected contexts as well as the positive samples of the real pairs of a response and its context. Note that it does not refer to ground-truth responses during the evaluations.

- RUBER is a blending metric between the referenced (Vector Pooling) and the unreferenced metric (NN Scorer) [Tao 17]. It outperforms the embedding-based and word-overlap-based metrics in many cases [Tao 17]. We followed the setting in a previous work [Tao 17] for training the NN scorer and inferring the score. We used both DailyDialog and SWBD to train the model.

- Entropy (Ent) is a diversity metric [Zhang 18] that reflects the evenness of the empirical n-gram distribution for the given responses:

$E n t=\frac{1}{\sum_{w \in \mathcal{V}} C(w)} \sum_{w \in \mathcal{V}} C(w) \log \frac{C(w)}{\sum_{w^{\prime} \in \mathcal{V}} C\left(w^{\prime}\right)}$, where $\mathcal{V}$ is a set of all n-grams in the given responses and $C(w)$ denotes the frequency of n-gram $w$. We set the 4-gram for evaluation.

- Controllability is the classification accuracy of the pre-trained dialogue act classifier determined using the training set*6 described in Section $4 \cdot 2$. We used the same dialogue act classifier. Any generated sentences are labeled by the classifier and compared with the given condition label to calculate the precision, recall, and $\mathrm{f} 1$.

\section{$5 \cdot 4$ Human Subjective Evaluation Metrics}

Since automatic evaluation scores suffer from a lack of correlation with human subjective evaluation results [Liu 16], We also examined models with a human subjective evaluation to confirm the naturalness and controllability of the generated responses on DailyDialog.

\section{\$1 Naturalness Evaluation}

We used a 3-point scale in accordance with an existing work [Xing 17] and randomly selected 240 generated responses from the test set. Human annotators added their evaluation scores for each sample by looking at the dialogue contexts. A detailed description follows:

*6 The accuracy of the classifiers was $81.59 \%$ (DailyDialog) and $87.27 \%$ (SWBD) in the test sets.
- +2: This response is not only relevant and natural but also informative and interesting.

- +1: This response can be used as a response in the context, although it is universal, like "Yes, I see," "Me too," or "I don 't know."

- 0: This response cannot be used in this context. It is either semantically irrelevant or disfluent.

Three annotators evaluated each sample, and their final score was decided by a simple majority. If all three evaluators completely disagreed $(0,+1$, and +2$)$, the example was scored as 1.

\section{\$ 2 Controllability Evaluation}

We manually evaluated whether the generated responses follow the given dialogue act labels. An expert annotator with three years of experience in dialogue act annotation categorized the dialogue acts for the generated responses. The annotator was trained using the training data of the DailyDialog corpus before the evaluation.

\section{Experimental Results}

\section{6-1 Automatic Evaluation Results}

\section{$\$ 1$ Overall Results}

Table 3 shows the results of the automatic evaluation. We compared our proposed models based on SeqGANs ( w/ IMPLICIT, w/ EXPLICIT, w/ ENSEMBLE) with the baseline models ( w/ SCE, w/ KgCVAE, w/ AC, w/ RL). $\overline{\text { Prec. }}$ and $\overline{\text { Rec. }}$ are the weighted averages ${ }^{* 7}$ of the precision/recall of each label of dialogue act.

For coherence, we confirmed that the models conditioned by the dialogue act labels (w/ SCE, w/ AC, w/ RL, w/ IMPLICIT, w/ EXPLICIT, w/ ENSEMBLE) improved the coherence scores (ROUGE-L, NN Scorer, and RUBER) compared to the models without conditions. In addition, the CHED models based on RL and SeqGANs achieved consistent improvement for each score compared with CHED w/ SCE. This result suggests that dialogue act conditions are a potential training constraint to improve the quality of the generated responses.

For controllability, CHED models based on RL and SeqGANs showed the improvement in controllability scores, although PPL was not worse than CHED w/ SCE. In particular, CHED w/ RL and CHED w/ ENSEMBLE showed the highest and second-highest improvement (over 90\%

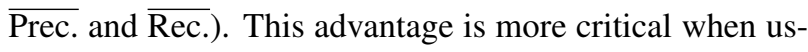
ing other decoding methods (e.g., random sampling and diverse beam search [Ippolito 18]). On the other hand, CHED w/ IMPLICIT's improvement was limited by a lack of explicit objectives to identify dialogue acts, unlike w/

\footnotetext{
*7 We use frequencies of each dialogue act label as weights.
} 
Table 3 Automatic evaluation results for each neural conversation model. Coherence and controllability scores are displayed on a scale from 0 to 100 .

\begin{tabular}{|c|c|c|c|c|c|c|c|c|c|}
\hline \multirow[b]{2}{*}{ Dataset } & \multirow[b]{2}{*}{ Models } & \multirow[b]{2}{*}{ PPL } & \multirow[b]{2}{*}{ Ent } & \multicolumn{4}{|c|}{ Coherence } & \multicolumn{2}{|c|}{ Controllability } \\
\hline & & & & ROUGE-L & Vector Pooling & NN Scorer & RUBER & $\overline{\text { Prec. }}$ & $\overline{\text { Rec. }}$ \\
\hline \multirow[t]{9}{*}{ DailyDialog } & בASEQ2SEQ & 34.97 & 10.90 & 15.31 & 84.04 & 46.46 & 42.52 & 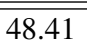 & 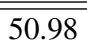 \\
\hline & HED & 33.03 & 9.59 & 13.79 & 83.87 & 47.12 & 43.08 & 46.90 & 48.83 \\
\hline & CHED w/ SCE & 32.11 & 10.16 & 16.66 & 83.94 & 46.11 & 42.40 & 86.65 & 86.01 \\
\hline & CHED w/ KgCVAE & 31.56 & 9.57 & 15.49 & 84.47 & 38.64 & 35.60 & 86.31 & 85.50 \\
\hline & $\mathrm{CHED}$ w/ AC & 32.74 & 10.07 & 17.03 & 83.97 & 47.03 & 43.09 & 87.72 & 87.86 \\
\hline & CHED w/ RL & 32.52 & 9.10 & 18.32 & 84.44 & 47.81 & 43.96 & 94.22 & 94.38 \\
\hline & CHED w/ IMPLICIT & 32.12 & 10.09 & 17.90 & 84.09 & 48.52 & 44.45 & 86.77 & 86.20 \\
\hline & CHED w/ EXPLICIT & 32.27 & 10.14 & 17.76 & 83.51 & 47.94 & 43.89 & 89.17 & 89.18 \\
\hline & CHED w/ ENSEMBLE & 32.30 & 10.31 & 17.98 & 83.53 & 48.39 & 44.32 & 90.51 & 90.52 \\
\hline \multirow[t]{9}{*}{ SWBD } & ASEQ2SEQ & 44.86 & 2.97 & 14.01 & 57.67 & 51.61 & 42.10 & 26.69 & 22.80 \\
\hline & HED & 44.30 & 3.32 & 16.17 & 59.18 & 54.86 & 44.17 & 46.62 & 31.31 \\
\hline & CHED w/ SCE & 41.18 & 4.92 & 26.20 & 71.78 & 51.22 & 45.59 & 92.14 & 74.82 \\
\hline & CHED w/ KgCVAE & 42.22 & 3.42 & 25.47 & 69.14 & 53.46 & 47.59 & 92.58 & 65.63 \\
\hline & $\mathrm{CHED}$ w/ AC & 44.05 & 4.81 & 25.22 & 70.33 & 54.77 & 48.01 & 93.87 & 74.95 \\
\hline & CHED w/ RL & 41.37 & 3.40 & 26.82 & 74.18 & 53.66 & 47.44 & 96.78 & 97.41 \\
\hline & CHED w/ IMPLICIT & 41.22 & 6.67 & 26.55 & 72.76 & 53.09 & 46.95 & 89.50 & 79.60 \\
\hline & CHED w/ EXPLICIT & 41.27 & 6.75 & 27.08 & 74.51 & 61.86 & 54.03 & 93.55 & 94.13 \\
\hline & CHED w/ ENSEMBLE & 41.29 & 5.70 & 26.97 & 74.21 & 62.41 & 54.46 & 95.20 & 95.79 \\
\hline
\end{tabular}

RL, w/ EXPLICIT, and w/ ENSEMBLE. CHED w/ KgCVAE did not show the improvement of controllability than CHED w/ SCE. This result is probably caused by the generation process of $\mathrm{KgCVAE}$, which strongly depends on sampling from latent variables. In other words, depending on the state of the hidden vectors sampled from the encoder network to generate the response, the discriminability of that generated response can vary greatly. CHED w/ AC showed higher controllability than CHED w/ SCE; however, the controllability was less than RL and SeqGANbased models. This is probably because of the sparsity of error signals from the discriminator when both the discriminator and generator over-fit the training data. Note that discriminability of CHED w/ KgCVAE and w/ AC, can be enhanced by applying our SeqGAN-based methods to decoder networks in fine-tuning after their training.

For diversity, the CHED models based on SeqGANs showed comparable or slight improvement compared to CHED w/ SCE. However, CHED w/ RL did not improve diversity, which especially decreased on SWBD. This suggests that CHED w/ RL generated responses that contain specific vocabulary or typical phrases.

\section{\$ 2 Controllability Results}

For a detailed analysis of the controllability of the response generation, we show the controllability score of CHED w/ ENSEMBLE for each dialogue act label in Table 4 (DailyDialog) and Table 5 (SWBD). The tables show the precision, recall, and the harmonic mean $(\mathrm{F} 1)$ of each dialogue act and the improvement from the score of CHED w/ SCE, which achieved the best controllability in the base- lines (improv.).

Table 4 Controllability for each dialogue act (DA) label on DailyDia$\log$ (CHED w/ ENSEMBLE vs. CHED w/ SCE)

\begin{tabular}{l||c|c|c|c}
\hline DA & Prec. & Rec. & F1 (improv.) & Freq. \\
\hline \hline Inform & 89.62 & 93.06 & $91.31(+\mathbf{3 . 7 5})$ & 3257 \\
\hline Questions & 98.89 & 99.24 & $99.07(+\mathbf{1 . 1 8})$ & 1713 \\
\hline Directives & 94.71 & 85.17 & $89.69(+\mathbf{8 . 8 4})$ & 1052 \\
\hline Commissive & 68.40 & 66.02 & $67.19(+\mathbf{7 . 9 6})$ & 718 \\
\hline
\end{tabular}

Our proposed CHED w/ ENSEMBLE improved the controllability of most classes, even if the target dialogue act labels have similar characteristics (e.g., "Directives" and "Commissive"). CHED w/ RL and CHED w/ EXPLICIT also showed a very similar improvement trend in the scores. However, we still must improve the controlled response generation based on minority dialogue act labels. We should not strongly accept the evaluation results from the dialogue act classifier, because annotating dialogue acts requires a great deal of expertise. Thus, a human expert annotator also evaluated the controllability of each model.

\section{$6 \cdot 2$ Results of Human Subjective Evaluation}

Table 6 and Table 7 show the human evaluation results for DailyDialog' s naturalness and controllability. Regarding the naturalness of the generated responses (Table 6), models based on SeqGANs generated more acceptable responses to the dialogue context. In particular, CHED w/ IMPLICIT achieved the highest performance, followed by CHED w/ ENSEMBLE. In contrast, HED w/ RL showed a slight decrease in overall naturalness despite being compa- 
Table 5 Controllability for each dialogue act (DA) label on SWBD (CHED w/ ENSEMBLE vs. CHED w/SCE). This table shows only dialogue act labels with a frequency of 5 or more.

\begin{tabular}{l||c|c|l|c}
\hline DA & Prec. & Rec. & F1 (improv.) & Freq. \\
\hline \hline $\begin{array}{l}\text { statement non } \\
\text { opinion }\end{array}$ & 95.14 & 99.22 & $97.13(+\mathbf{0 . 9 7})$ & 2169 \\
\hline $\begin{array}{l}\text { acknowledgements } \\
\text { (backchannel) }\end{array}$ & 97.17 & 98.88 & $98.02(+\mathbf{0 . 9 6})$ & 1250 \\
\hline statement opinion & 97.88 & 86.94 & $92.08(+\mathbf{8 . 4 5})$ & 689 \\
\hline $\begin{array}{l}\text { abandoned turned } \\
\text { or } \\
\text { exit/uninterpretable }\end{array}$ & 99.41 & 100.00 & $99.70(+\mathbf{0 . 4 4})$ & 674 \\
\hline yes/no question & 93.96 & 97.90 & $95.89(+\mathbf{1 8 . 7 6})$ & 143 \\
\hline appreciation & 96.40 & 100.00 & $98.17(-0.34)$ & 134 \\
\hline agree/accept & 84.69 & 80.58 & $82.59(+\mathbf{7 1 . 2 4})$ & 103 \\
\hline yes answers & 89.47 & 65.38 & $75.56(+\mathbf{3 . 6 5})$ & 52 \\
\hline wh question & 78.46 & 100.00 & $87.93(-8.14)$ & 51 \\
\hline $\begin{array}{l}\text { backchannel in } \\
\text { question form }\end{array}$ & 100.00 & 100.00 & $100.00(+\mathbf{1 . 1 5})$ & 43 \\
\hline $\begin{array}{l}\text { conventional clos- } \\
\text { ing }\end{array}$ & 97.56 & 100.00 & $98.77(+\mathbf{3 . 6 5})$ & 40 \\
\hline $\begin{array}{l}\text { response acknowl- } \\
\text { edgement }\end{array}$ & 94.74 & 100.00 & $97.30(+\mathbf{3 7 . 3 0})$ & 18 \\
\hline open question & 66.67 & 100.00 & $80.00(+\mathbf{5 . 0 0})$ & 18 \\
\hline no answers & 78.57 & 73.33 & $75.86(+\mathbf{1 6 . 4 0})$ & 15 \\
\hline $\begin{array}{l}\text { declarative yes/no } \\
\text { question }\end{array}$ & 100.00 & 10.00 & $18.18(+0.00)$ & 10 \\
\hline $\begin{array}{l}\text { affirmative non- } \\
\text { yes answers }\end{array}$ & 50.00 & 75.00 & $60.00(+\mathbf{2 2 . 1 5})$ & 8 \\
\hline $\begin{array}{l}\text { rhetorical ques- } \\
\text { tions }\end{array}$ & 0.00 & 0.00 & $0.00(+0.00)$ & 8 \\
\hline $\begin{array}{l}\text { summarize or re- } \\
\text { formulate }\end{array}$ & 100.00 & 25.00 & $40.00(+\mathbf{4 0 . 0 0 )}$ & 8 \\
\hline action directive & 0.00 & 0.00 & $0.00(-60.00)$ & 7 \\
\hline other & 100.00 & 33.33 & $50.00(+\mathbf{5 . 5 6})$ & 6 \\
\hline
\end{tabular}

rable to models based on SeqGANs in the automatic evaluations.

Table 6 Response quality of each model with different objective functions in human evaluations. This table shows percentages for each score. Avg is weighted by percentage of scores.

\begin{tabular}{l||c|c|c|c}
\hline Models & +2 & +1 & +0 & Avg \\
\hline \hline HED w/ SCE & 23.33 & 38.33 & 38.33 & 0.85 \\
\hline CHED w/ SCE & 25.00 & 38.75 & 36.25 & 0.88 \\
\hline CHED w/ RL & 24.58 & 36.66 & 38.75 & 0.85 \\
\hline CHED w/ IMPLICIT & 27.91 & 43.33 & 28.75 & $\mathbf{0 . 9 9}$ \\
\hline CHED w/ EXPLICIT & 25.41 & 39.58 & 35.00 & 0.90 \\
\hline CHED w/ ENSEMBLE & 27.91 & 41.25 & 30.83 & 0.97 \\
\hline
\end{tabular}

Regarding the controllability of the generated responses (Table 7), CHED w/ EXPLICIT and CHED w/ ENSEM-

BLE achieved the highest and second-highest performances. CHED w/ RL showed slightly inferior or comparable performance to CHED w/ EXPLICIT and CHED w/ ENSEMBLE, although CHED w/ RL remarkably improved controllability compared with the other models in the automatic evaluation. This is because the evaluation result of the dialogue act classifier used as a reward function on RL does not always agree with human evaluations. In other words, the CHED w/ RL model may be over-fitted to the dialogue act classifier. It learns strategies that is over-fitted to the dialogue act classifier, and the naturalness of the generated responses is only considered by the SCE-loss used as an auxiliary objective. In contrast, our models using the explicit-discriminator, which is dynamically updated by adversarial learning, have the potential to generate more natural and discriminable responses.

Table 7 Controllability of response generation: We calculated agreement (Cohen $\mathrm{s} \kappa$ ) with automatic annotation results by dialogue act classifier. Table shows the filtered results of contradicted responses. Ratio is the proportion of the acceptable response that was properly controlled by dialogue acts.

\begin{tabular}{l||c|c|c}
\hline Models & Accuracy & Agreement & Ratio \\
\hline \hline CHED w/ SCE & 85.14 & 0.68 & 54.27 \\
\hline CHED w/ RL & 91.54 & 0.82 & 56.06 \\
\hline CHED w/ IMPLICIT & 87.84 & 0.72 & 62.58 \\
\hline CHED w/ EXPLICIT & $\mathbf{9 3 . 0 6}$ & 0.69 & 60.48 \\
\hline CHED w/ ENSEMBLE & 92.68 & 0.81 & $\mathbf{6 4 . 1 2}$ \\
\hline
\end{tabular}

These results suggest that the implicit-discriminator improves the naturalness, and the explicit-discriminator improves the controllability in our adversarial learning. Furthermore, the ensemble approach, which combines both discriminators, has the potential to produce better naturalness and controllability results.

\subsection{Dialogue Examples}

We show examples generated by our models in Table 8 . Our proposed CHED w/ ENSEMBLE generated a comparable or slightly natural response compared to other models, even though their responses represent the characteristics of dialogue act labels given during response generation. Here, note that the outputs of the listed models are very similar in some cases (e.g., first and second examples). This is because CHED w/ SCE is shared as the pre-trained model for each model. Hence, if the possible responses that CHED w/ SCE might generate for a given context are already appropriate, or if enough search in RL is not performed, the response generation's tendency may not vary significantly in case.

\section{Conclusion}

We introduced an extended framework of sequential generative adversarial networks to improve the controllability of neural conversation models (NCMs) under the constraint of a given dialogue act label. We propose a different kind of adversarial approach, including a new label-aware objective to encourage the generation of discriminative responses by given dialogue act labels. Experimental results showed that our proposed method, which is based on an 
Table 8 Generated responses from models given a dialogue history and a dialogue act. A and B denote system and user. Reference utterances for dialogue context sampled from test set shown after the context. Generation results in contexts from models are shown after references. Before generation results, given dialogue acts are indicated by round brackets.

\begin{tabular}{|c|c|}
\hline $\begin{array}{l}\text { A } \\
\text { B } \\
\text { A: Human } \\
\text { A: HED } \\
\text { A: CHED } \\
\text { A: CHED w/ RL } \\
\text { A: CHED w/ IMPLICIT } \\
\text { A: CHED w/ EXPLICIT } \\
\text { A: CHED w/ ENSEMBLE }\end{array}$ & $\begin{array}{l}\text { (Commissive) yes of course . this is a really good one actually. } \\
\text { (Questions) how much does it cost ? } \\
\text { (Inform) this one is } 1500 \text { yuan . } \\
\text { (Inform) want to take a look at it. } \\
\text { (Inform) \$200, please. } \\
\text { (Inform) \$ } 800 \text { per month. } \\
\text { (Inform) \$200, please. } \\
\text { (Inform) \$200. } \\
\text { (Inform) \$200, please. }\end{array}$ \\
\hline $\begin{array}{l}\text { A } \\
\text { B } \\
\text { A: Human } \\
\text { A: HED } \\
\text { A: CHED } \\
\text { A: CHED w/ RL } \\
\text { A: CHED w/ IMPLICIT } \\
\text { A: CHED w/ EXPLICIT } \\
\text { A: CHED w/ ENSEMBLE }\end{array}$ & $\begin{array}{l}\text { (Questions) what's the problem , nada? you look down in the dumps . } \\
\text { (Inform) i don t know . my life is a big mess . everything is so complicated } \\
\text { (Questions) come on , nothing can be that bad? } \\
\text { (Questions) what do you think is the most important thing for you? } \\
\text { (Questions) what do you mean? } \\
\text { (Questions) what do you think of the most important thing in the city? } \\
\text { (Questions) what do you mean? } \\
\text { (Questions) what do you mean? } \\
\text { (Questions) what do you think is the most important thing for you to do? }\end{array}$ \\
\hline $\begin{array}{l}\text { A } \\
\text { B } \\
\text { A: Human } \\
\text { A: HED } \\
\text { A: CHED } \\
\text { A: CHED w/ RL } \\
\text { A: CHED w/ IMPLICIT } \\
\text { A: CHED w/ EXPLICIT } \\
\text { A: CHED w/ ENSEMBLE }\end{array}$ & $\begin{array}{l}\text { (Inform) it 's getting late . i really have to go . } \\
\text { (Directives) i hope we' } 11 \text { get together again soon . } \\
\text { (Commissive) i hope so . a good journey to you! } \\
\text { (Commissive) i don, t know what to do . } \\
\text { (Commissive) i don t think so. } \\
\text { (Commissive) ok. let , s go. } \\
\text { (Commissive) ok. let s go . } \\
\text { (Commissive) i will. thank you for your help. } \\
\text { (Commissive) i think so }\end{array}$ \\
\hline $\begin{array}{l}\text { A } \\
\text { B: Human } \\
\text { B: HED } \\
\text { B: CHED } \\
\text { B: CHED w/ RL } \\
\text { B: CHED w/ IMPLICIT } \\
\text { B: CHED w/ EXPLICIT } \\
\text { B: CHED w/ ENSEMBLE }\end{array}$ & $\begin{array}{l}\text { (Directives) i d like to make an appointment with dr. cooper . could you arrange it for me ? } \\
\text { (Directives) yes . would tomorrow morning be all right with you ? } \\
\text { (Directives) what about this one ? } \\
\text { (Directives) i need to make a reservation for me , please . } \\
\text { (Directives) i need to check out this form . can you give me a call , please? } \\
\text { (Directives) sure. would you please fill out this form , please? } \\
\text { (Directives) well , i would like to buy a ticket . } \\
\text { (Directives) you need to check out this form . would you like to go with me? }\end{array}$ \\
\hline
\end{tabular}

ensemble rewarding strategy, has higher controllability for dialogue acts even though it has higher or comparable naturalness to existing methods.

However, the response quality of our proposal still has room for improvement. According to the human evaluation results, $70 \%$ of the generated responses did not cause dialogue breakdowns. However, their responses remain inadequate to satisfy the user. It is difficult to continue multi-turn conversations due to the limitation of the training data in the available dataset, such as DailyDialog and SWBD corpus. Thus, we need to explore an approach to address this problem. Exploring a method to control the response of NCMs based on BERT [Devlin 19] or DialoGPT [Zhang 20], which are pre-trained on massive dialogue corpora, is one promising direction. Furthermore, we will integrate the optimization of natural language understanding and dialogue management into our framework while focusing on different types of labels (e.g., emotions and persona) for building a more user-satisfied conditional NCM.

\section{Acknowledgments}

This work was supported by JSPS under Grant 20J14823.

\section{$\diamond$ References $\diamond$}

[Alston 00] Alston, W. P.: Illocutionary Acts and Sentence Meaning, Cornell University Press (2000)

[Boyer 10] Boyer, K., Ha, E. Y., Phillips, R., Wallis, M., Vouk, M., and Lester, J.: Dialogue act modeling in a complex task-oriented domain, in Proceedings of the SIGDIAL 2010 Conference, pp. 297305 (2010)

[Bunt 12] Bunt, H., Alexandersson, J., Choe, J.-W., Fang, A. C., Hasida, K., Petukhova, V., Popescu-Belis, A., and Traum, D. R.: ISO 24617-2: A semantically-based standard for dialogue annotation, in Proceedings of Conference on International Language Resources and Evaluation, pp. 430-437 (2012)

[Cho 14] Cho, K., Merrienboer, van B., Gulcehre, C., Bahdanau, D., Bougares, F., Schwenk, H., and Bengio, Y.: Learning phrase representations using RNN encoder-decoder for statistical machine translation, in Proceedings of EMNLP, pp. 1724-1734 (2014)

[Devlin 19] Devlin, J., Chang, M.-W., Lee, K., and Toutanova, K.: BERT: Pre-training of deep bidirectional transformers for language understanding, in Proceedings of the 2019 Conference of the North American Chapter of the Association for Computational Linguistics: Human Language Technologies, Volume 1 (Long and Short Papers), pp. 4171-4186 (2019)

[Dušek 18] Dušek, O., Novikova, J., and Rieser, V.: Findings of the E2E NLG challenge, in Proceedings of the 11th International Conference on Natural Language Generation, pp. 322-328 (2018)

[Hu 17] Hu, Z., Yang, Z., Liang, X., Salakhutdinov, R., and Xing, E. P.: Toward controlled generation of text, in International Conference on Machine Learning, pp. 1587-1596 (2017)

[Huang 18] Huang, C., Zaiane, O. R., Trabelsi, A., and Dziri, N.: Automatic dialogue generation with expressed emotions, in Proceedings of the 2018 Conference of the North American Chapter of the Association for Computational Linguistics: Human Language Tech- 
nologies, Volume 2 (Short Papers), pp. 49-54 (2018)

[Ippolito 18] Ippolito, D., Kriz, R., Sedoc, J., Kustikova, M., Callison-Burch, C., Kriz, R., Miltsakaki, E., Apidianaki, M., Callison-Burch, C., Hewitt, J., et al.: Comparison of Diverse Decoding Methods from Conditional Language Models, in Proceedings of the 57th Conference of the Association for Computational LinguisticsAssociation for Computational Linguistics (2018)

[Jang 16] Jang, E., Gu, S., and Poole, B.: Categorical reparameterization with gumbel-softmax, arXiv preprint arXiv:1611.01144 (2016)

[Jurafsky 97] Jurafsky, D.: Switchboard SWBD-DAMSL shallowdiscourse-function annotation coders manual, www. dcs. shef. ac. uk/nlp/amities/files/bib/ics-tr-97-02. pdf (1997)

[Kawano 19] Kawano, S., Yoshino, K., and Nakamura, S.: Neural Conversation Model Controllable by Given Dialogue Act Based on Adversarial Learning and Label-aware Objective, in Proceedings of the 12th International Conference on Natural Language Generation (2019)

[Keizer 17] Keizer, S. and Rieser, V.: Towards learning transferable conversational skills using multi-dimensional dialogue modelling, in Proceedings of SEMDIAL 2017 (SaarDial) Workshop on the Semantics and Pragmatics of Dialogue, pp. 150-151 (2017)

[Kingma 14] Kingma, D. P. and Ba, J.: Adam: A method for stochastic optimization, arXiv preprint arXiv: 1412.6980 (2014)

[Kong 19] Kong, X., Li, B., Neubig, G., Hovy, E., and Yang, Y.: An adversarial approach to high-quality, sentiment-controlled neural dialogue generation, in AAAI 2019 Workshop on Reasoning and Learning for Human-Machine Dialogues (DEEP-DIAL 2019), Honolulu, Hawaii (2019)

[Li 16a] Li, J., Galley, M., Brockett, C., Gao, J., and Dolan, W. B.: A diversity-promoting objective function for neural conversation models, in Proceedings of the 2016 Conference of the North American Chapter of the Association for Computational Linguistics: Human Language Technologies, pp. 110-119 (2016)

[Li 16b] Li, J., Galley, M., Brockett, C., Spithourakis, G. P., Gao, J., and Dolan, B.: A persona-based neural conversation model, arXiv preprint arXiv:1603.06155 (2016)

[Li 17a] Li, J., Monroe, W., Shi, T., Jean, S., Ritter, A., and Jurafsky, D.: Adversarial Learning for Neural Dialogue Generation, in Proceedings of the 2017 Conference on Empirical Methods in Natural Language Processing, pp. 2157-2169 (2017)

[Li 17b] Li, Y., Su, H., Shen, X., Li, W., Cao, Z., and Niu, S.: DailyDialog: A manually labelled multi-turn dialogue dataset, arXiv preprint arXiv:1710.03957 (2017)

[Lin 04] Lin, C.-Y. and Och, F. J.: Automatic evaluation of machine translation quality using longest common subsequence and skipbigram statistics, in Proceedings of the 42nd Annual Meeting of the Association for Computational Linguistics (ACL-04), pp. 605-612 (2004)

[Liu 16] Liu, C.-W., Lowe, R., Serban, I. V., Noseworthy, M., Charlin, L., and Pineau, J.: How Not to evaluate your dialogue system: An empirical study of unsupervised evaluation metrics for dialogue response generation, in Proceedings of the 2016 Conference on Empirical Methods in Natural Language Processing, pp. 2122-2132 (2016)

[Luong 15] Luong, M.-T., Pham, H., and Manning, C. D.: Effective approaches to attention-based neural machine translation, arXiv preprint arXiv:1508.04025 (2015)

[Meguro 10] Meguro, T., Higashinaka, R., Minami, Y., and Dohsaka, K.: Controlling listening-oriented dialogue using partially observable Markov decision processes, in Proceedings of the 23rd International Conference on Computational Linguistics (Coling 2010), pp. 761-769 (2010)

[Odena 16] Odena, A.: Semi-supervised learning with generative adversarial networks, arXiv preprint arXiv:1606.01583 (2016)

[Oh 00] Oh, A. H. and Rudnicky, A. I.: Stochastic language generation for spoken dialogue systems, in ANLP-NAACL 2000 Workshop: Conversational Systems (2000)

[Peng 19] Peng, Y., Fang, Y., Xie, Z., and Zhou, G.: Topicenhanced emotional conversation generation with attention mechanism, Knowledge-Based Systems, Vol. 163, pp. 429-437 (2019)

[Ranzato 16] Ranzato, M., Chopra, S., Auli, M., and Zaremba, W.:
Sequence Level Training with Recurrent Neural Networks, in Bengio, Y. and LeCun, Y. eds., in Proceedings of the 4th International Conference on Learning Representations (2016)

[Salimans 16] Salimans, T., Goodfellow, I., Zaremba, W., Cheung, V., Radford, A., and Chen, X.: Improved techniques for training gans, in Advances in Neural Information Processing Systems, pp. 2234-2242 (2016)

[Serban 16] Serban, I., Sordoni, A., Bengio, Y., Courville, A., and Pineau, J.: Building end-to-end dialogue systems using generative hierarchical neural network models, in Proceedings of the AAAI Conference on Artificial Intelligence, Vol. 30 (2016)

[Shen 20] Shen, L. and Feng, Y.: CDL: Curriculum dual learning for emotion-controllable response generation, in Proceedings of the 58th Annual Meeting of the Association for Computational Linguistics, pp. 556-566, Association for Computational Linguistics (2020)

[Shibata 16] Shibata, T., Egashira, Y., and Kurohashi, S.: Chat-like conversational system based on selection of reply generating module with reinforcement learning, in Situated Dialog in Speech-Based Human-Computer Interaction, pp. 63-69, Springer (2016)

[Stolcke 00] Stolcke, A., Ries, K., Coccaro, N., Shriberg, E., Bates, R., Jurafsky, D., Taylor, P., Martin, R., Van Ess-Dykema, C., and Meteer, M.: Dialogue act modeling for automatic tagging and recognition of conversational speech, Computational Linguistics, Vol. 26, No. 3, pp. 339-373 (2000)

[Sun 18] Sun, X., Chen, X., Pei, Z., and Ren, F.: Emotional human machine conversation generation based on SeqGAN, in 2018 First Asian Conference on Affective Computing and Intelligent Interaction (ACII Asia), pp. 1-6 (2018)

[Tao 17] Tao, C., Mou, L., Zhao, D., and Yan, R.: Ruber: An unsupervised method for automatic evaluation of open-domain dialog systems, arXiv preprint arXiv:1701.03079 (2017)

[Tuan 19] Tuan, Y.-L. and Lee, H.-Y.: Improving conditional sequence generative adversarial networks by stepwise evaluation, IEEE/ACM Transactions on Audio, Speech, and Language Processing (2019)

[Vinyals 15] Vinyals, O. and Le, Q.: A neural conversational model, arXiv preprint arXiv: 1506.05869 (2015)

[Wen 15] Wen, T.-H., Gasic, M., Mrksic, N., Su, P.-H., Vandyke, D., and Young, S.: Semantically conditioned lstm-based natural language generation for spoken dialogue systems, arXiv preprint arXiv:1508.01745 (2015)

[Williams 92] Williams, R. J.: Simple statistical gradient-following algorithms for connectionist reinforcement learning, Machine Learning, Vol. 8, No. 3-4, pp. 229-256 (1992)

[Xing 17] Xing, C., Wu, W., Wu, Y., Liu, J., Huang, Y., Zhou, M., and Ma, W.-Y.: Topic aware neural response generation, in 31st AAAI Conference on Artificial Intelligence (2017)

[Xu 00] Xu, W. and Rudnicky, A. I.: Task-based dialog management using an agenda, in ANLP-NAACL 2000 Workshop: Conversational Systems (2000)

[Yoshino 15] Yoshino, K. and Kawahara, T.: Conversational system for information navigation based on POMDP with user focus tracking, Computer Speech \& Language, Vol. 34, No. 1, pp. 275-291 (2015)

[Young 10] Young, S., Gašić, M., Keizer, S., Mairesse, F., Schatzmann, J., Thomson, B., and Yu, K.: The hidden information state model: A practical framework for POMDP-based spoken dialogue management, Computer Speech \& Language, Vol. 24, No. 2, pp. 150-174 (2010)

[Yu 17] Yu, L., Zhang, W., Wang, J., and Yu, Y.: Seqgan: Sequence generative adversarial nets with policy gradient, in Proceedings of the AAAI Conference on Artificial Intelligence, Vol. 31 (2017)

[Zhang 18] Zhang, Y., Galley, M., Gao, J., Gan, Z., Li, X., Brockett, C., and Dolan, B.: Generating informative and diverse conversational responses via adversarial information maximization, in $\mathrm{Ad}$ vances in Neural Information Processing Systems, pp. 1810-1820 (2018)

[Zhang 20] Zhang, Y., Sun, S., Galley, M., Chen, Y.-C., Brockett, C., Gao, X., Gao, J., Liu, J., and Dolan, B.: DIALOGPT : Large-scale generative pre-training for conversational response generation, in Proceedings of the 58th Annual Meeting of the Association for Com- 
putational Linguistics: System Demonstrations, pp. 270-278 (2020)

[Zhao 17] Zhao, T., Zhao, R., and Eskenazi, M.: Learning discourselevel diversity for neural dialog models using conditional variational autoencoders, in Proceedings of the 55th Annual Meeting of the Association for Computational Linguistics (Volume 1: Long Papers), pp. 654-664 (2017)

[Zhou 18a] Zhou, H., Huang, M., Zhang, T., Zhu, X., and Liu, B.: Emotional chatting machine: Emotional conversation generation with internal and external memory, in 32nd AAAI Conference on Artificial Intelligence (2018)

[Zhou 18b] Zhou, X. and Wang, W. Y.: MojiTalk: Generating emotional responses at scale, in Proceedings of the 56th Annual Meeting of the Association for Computational Linguistics (Volume 1: Long Papers), pp. 1128-1137 (2018)

[担当委員：井上 昂治]

Received December 28, 2020.

\section{Author's Profile}

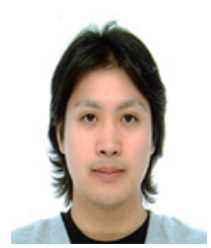

\section{Seiya Kawano (Student Member)}

He received the B.E. degree from the National Institution for Academic Degrees and Quality Enhancement of Higher Education, in 2016, and the M.E. degree from the Nara Institute of Science and Technology (NAIST), Japan, in 2018, where he is currently pursuing the Ph.D. degree. He was also a Research Fellow (DC2) of the Japan Society for the

Promotion of Science from 2020 to 2021. Currently, he is a research associate of Guardian Robot Project (GRP) at Institute of Physical and Chemical Research (RIKEN). His research interest includes natural language processing, especially dialogue systems. He is a member of ACL.

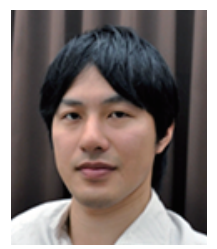

Koichiro Yoshino (Member)

He is a Team Leader of Guardian Robot Project (GRP) at Institute of Physical and Chemical Research (RIKEN), and a Visiting Associate Professor at Nara Institute of Science and Technology (NAIST). He received his bachelor degree of arts from Keio University in 2009, master degree of informatics from Kyoto University in 2011, and Ph.D. in informatics from Kyoto University in 2014. He worked at Kyoto University as a postdoc, and NAIST as an assistant professor. He is working on areas of spoken and natural language processings, especially on robot dialogue systems. He received several honors including the best paper award of IWSDS 2020, and the best paper award of the 1st NLP4ConvAI workshop. He is a member of IEEE, SIGDIAL, ACL, ANLP, IPSJ and RSJ.

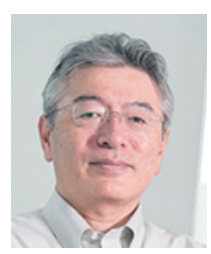

\section{Satoshi Nakamura (Member)}

$\mathrm{He}$ is a professor at the Nara Institute of Science and Technology, Team Leader of the Tourism Information Analytics Team, AIP Center, RIKEN, and Honorary professor at the Karlsruhe Institute of Technology, Germany. He received his B.S. from the Kyoto Institute of Technology in 1981 and a Ph.D. from the Kyoto University in 1992 . He was the Director of ATR Spoken Language Communication Research Laboratories in the period 2000-2008 and Vice President of ATR in the period 2007-2008. He was the Director General of Keihanna Research Laboratories and Executive Director of Knowledge Creating Communication Research Center, National Institute of Information and Communications Technology in 2009-2010. He is currently the director of the Augmented Human Communication laboratory and a full professor at the Data Science Center and Information Science Division, Graduate School of Science and Technology, Nara Institute of Science and Technology. He is one of the leaders of speech-to-speech translation research and has been serving various speech-to-speech translation research projects in the world, including C-STAR, IWSLT, and A-STAR. His research interests include the modeling and systems of speech-to-speech translation and spoken language processing. He was an Elected Board Member of the International Speech Communication Association, ISCA, in the period June 2011-2019, and IEEE Signal Processing Magazine Editorial Board member in 2012-2015, IEEE SPS Speech and Language Technical Committee Member in 2013-2015. He is an ATR Fellow, IPSJ Fellow, IEEE Fellow, and ISCA Fellow. 